IVYSPRING

INTERNATIONAL PUBLISHER

\section{Theranostics}

2012; 2(4):403-412. doi: 10.7150/thno.3634

Review

\title{
Magnetic Resonance Reporter Gene Imaging
}

\author{
Sheen-Woo Lee ${ }^{1 凶}$, Sang-Hoon Lee ${ }^{2}$, Sandip Biswal ${ }^{3}$
}

1. Department of Radiology, Gil Hospital, Gachon University School of Medicine and Science, Inchon, Republic of Korea

2. Department of Radiology, Asan Medical Center, Ulsan University College of Medicine, Seoul, Republic of Korea

3. Department of Radiology, Stanford University School of Medicine, Stanford, United States

Corresponding author: leesw1@gilhospital.com. 1198 Guwol-dong, Namdong-gu, Inchon, Republic of Korea. TEL +82-2-32-460-3066, FAX +82-2-32-460-3065

(C) Ivyspring International Publisher. This is an open-access article distributed under the terms of the Creative Commons License (http://creativecommons.org/ licenses/by-nc-nd/3.0/). Reproduction is permitted for personal, noncommercial use, provided that the article is in whole, unmodified, and properly cited.

Received: 2011.10.12; Accepted: 2012.03.09; Published: 2012.04.12

\begin{abstract}
Molecular imaging has undergone an explosive advancement in recent years, due to the tremendous research efforts made to understand and visualize biological processes. Molecular imaging by definition assesses cellular and molecular processes in living subjects, with the targets of following metabolic, genomic, and proteomic events. Furthermore, reporter gene imaging plays a central role in this field. Many different approaches have been used to visualize genetic events in living subjects, such as, optical, radionuclide, and magnetic resonance imaging. Compared with the other techniques, magnetic resonance (MR)-based reporter gene imaging has not occupied center stage, despite its superior three-dimensional depictions of anatomical details. In this article, the authors review the principles and applications of various types of MR reporter gene imaging technologies and discuss their advantages and disadvantages.
\end{abstract}

Key words: Magnetic resonance imaging, Reporter gene, molecular imaging.

\section{Principles of molecular magnetic resonance imaging}

The major goal of molecular imaging is the spatiotemporal imaging of genomic and proteomic events. Molecular imaging encompasses the fields of chemistry, biology, physics, and medicine, and brings experts in these scientific fields together to determine means of visualizing molecular and cellular events [1, 2]

Molecular imaging includes several imaging modalities, such as, bioluminescence, fluorescence, positron emission tomography (PET), single photon emission computed tomography (SPECT), computed tomography (CT), optical imaging, ultrasound, and magnetic resonance imaging (MRI). The advantages and disadvantages of these modalities have been discussed extensively in the literature [1]. MRI is well known for its superior three-dimensional resolution, and can be used to acquire physiological and ultra-fine anatomical information using different pulse sequences. Unlike other cross-sectional modalities, such as, PET, SPECT, and CT, MRI is free from the issue of ionizing radiation with arbitrary imaging planes and provides multiplanar imaging capabilities. Ultrasound and optical imaging are limited in their ability to detect signals through deep tissue. CT provides anatomical maps for PET/SPECT; however, its role in molecular imaging is restricted due to its low sensitivity and limited contrast resolution of soft tissue. One of the biggest advantages of MRI is its ability to provide images of deep tissues in a background of superb anatomical detail. Furthermore like CT and ultrasound, MR scanners are widely available that can accommodate large animals.

The disadvantages of MRI are difficult interpre- 
tation among complex background signal intensity and its relatively low sensitivity. According to Massoud et al, the sensitivity of MRI probe detection is $10^{6}-10^{9}$ times lower than PET and $10^{10}-10^{14}$ times lower than bioluminescence. However, thanks to the development of high field MRI and improved hardware and software designs, the signal to noise ratio of MRI has been improved significantly [1], and novel approaches used for reporter gene imaging may further increase its sensitivity and specificity.

In a typical MRI, the signal provided by the smallest image element is a function of mobile proton in hydrogen molecule and the relaxation time. There are two types of relaxation times, that is, a T1 spin-lattice relaxation time, and a T2 spin-spin relaxation time. Different pulse sequences exploit localized variations in these relaxation times, so that a given element (a voxel) takes on different signal intensities according to physiological conditions. "Enhancement" is used to increase voxel contrast by perturbing the local environment. Gadolinium, the most commonly used MR contrast "enhancement" material, shortens T1 and produces bright voxels corresponding to gadolinium-containing region on T1-weighted images. On the other hand, iron, the second most common contrast agent, shortens $\mathrm{T} 2$, and produces dark voxels on T2-weighted images. T1-shortening agents are more useful clinically because target lesions are imaged at high signal-to-background ratios, whereas T2-shortening agents have higher sensitivity at the molecular level [3] .

Two types of labeling mechanisms can be used to target a biological process or a cell using MRI. Direct labeling involves the binding of gadolinium or an iron-containing compound to the cell surface or internalized intracellularly. The other mechanism involves reporter gene techniques that induce specific genetic cascades. The major benefits of reporter gene techniques are that cells must be viable to produce an imaging signal, and that imaging signal does not dilute with cell division [4]. Research is being undertaken to find safe transgene strategies for reporter gene imaging.

\section{The mechanism of MR reporter gene imaging}

Reporter gene imaging by MRI can be grouped into four types: enzyme-based, spectroscopy-based, iron-related, or chemical exchange saturation transfer (CEST)-based.

\section{Enzyme-based Studies}

Enzyme-based MR can be divided into two modalities, one for MR spectroscopy (MRS) and the other for MR imaging (MRI).
Several approaches to enzyme-based MRI have been devised. One pioneering example involved the use of B-galactosidase (Figure 1). Louie et al. developed a gadolinium-based substrate that contains a galactose group, which conceals the central gadolinium atom. In the presence of galactosidase (introduced by lac $Z$ transfection), the galactopyranose moiety is enzymatically cleaved, which allows a water molecule to access the gadolinium, and increase the T1 signal [5]. More recent study combined lacZ-transfected tumor with 3,4-cyclohexenoesculetin b-D-galactopyranoside and ferric ion, which result in T2* relaxation on MRI [6].
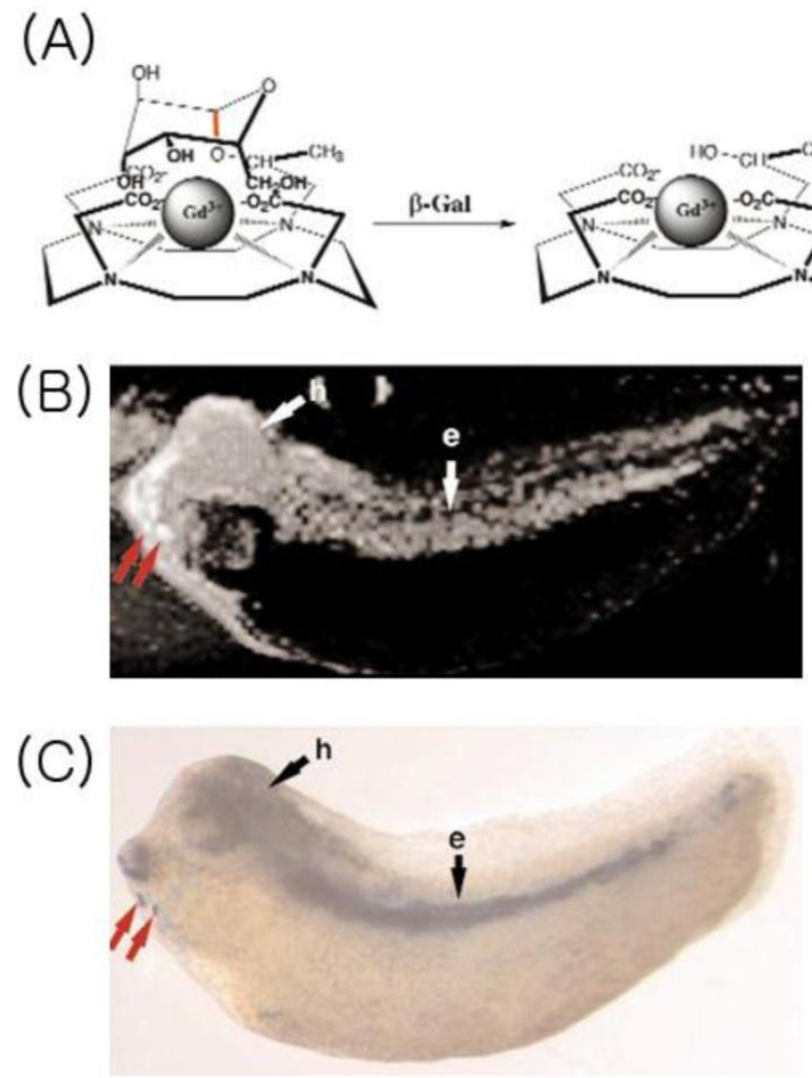

Figure I. EgadMe. (A) Schematic diagram representing the site-specific placement of the galactopyranosyl ring on the tetra-azamacrocycle. When galactosidase cleaves the sugar (at the red bond), the inner $\mathrm{Gd}^{3+}$ ion becomes more accessible to water. (B) 3-dimensional TI-weighted MR image of a living embryo. Plasmid carrying the lac $Z$ gene was injected into one cell at the two-cell stage.Subsequent enzyme expression was on the left side of the embryo. Regions of high signal intensity were found in the endoderm (e), head (h), and ventrally (red arrows). (C) Bright-field image of an embryo fixed and stained with X-gal. Regions demonstrating enzyme expression correlated with regions of high intensity in MR images. [Reprinted from ref. 5 with permission]. 


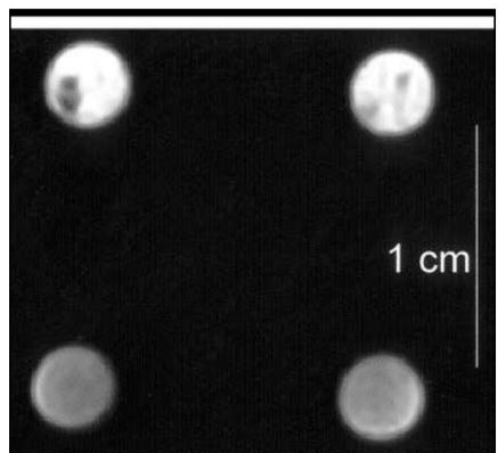

Figure 2. TI-weighted gradient-echo MR image of cells with or without tyrosinase. Image of two cell clones (one clone in each column) cultured under conditions that allowed tyrosinase expression (top row) or did not allow its expression (bottom row). After culturing with iron- and holotransferrin-enriched medium, cells that were tyrosinase positive showed higher signal intensity by TIWI imaging because of the presence of metallomelanin. [Reprinted from ref. 8 with permission].

Another example is provided by the tyrosinase gene (Figure 2). Tyrosinase is required for the production of melanin, which binds paramagnetic iron ions to produce metallomelanin, a T1-shortening agent. Cells overexpressing tyrosinase thus exhibit high signal intensity on T1-weighted images [7, 8].

Nevertheless, enzyme-based MR reporter gene imaging faces many challenges. One problem associated with the $B$-gal-based approach is that even when the enzyme has not cleaved off galactose, outer surface relaxation changes of the gadolinium-containing compound are non-zero. Thus, while one might assume that the background signal would be zero without any endogenous B-galactosidase, some enhancement may actually occur in practice. Another problematic issue concerns the delivery barrier, which restricts substrate access to the transfected cell. In addition, the persistence of melanin and metallomelanin in cells, even when the reporter gene is not activated, cause false high MRI signal intensities [9].

\section{Spectroscopy-based Studies}

MR spectroscopy or MRS techniques take use catalytic enzymes, such as, creatine kinase and arginine kinase, and their conversion of ATP to ADP, using 31P MR spectroscopy (MRS) [10]. For example, the arginine kinase gene from Drosophila can be transfected into vertebrate muscle using a viral vector, and the byproduct of the enzymatic conversion of ATP, phosphorarginine, can be detected by 31P MRS. The expression and activity of the transfected kinase can be followed in living mammalian muscle for several months after injection [11]. Another approach involves the use of a reporter enzyme to follow a pro- drug during its internalization and conversion by a target cell, for example, the cytosine deaminase gene catalyzes the conversion of the prodrug 5-fluorocytosine into 5-fluorouracile in tumor cells, which can be monitored by 19F MRS [12]. The fluorouracile and fluorocytosine, being anions, cannot cross the cell membrane and are retained in the cell, therefore serve as a means to develop contrast. Furthermore, the cytosine deaminase result in toxic metabolite formation in the transfected tumor, suggesting that reporter gene technology can provide cancer gene therapy in the future [12-14].

\section{OFPNP}

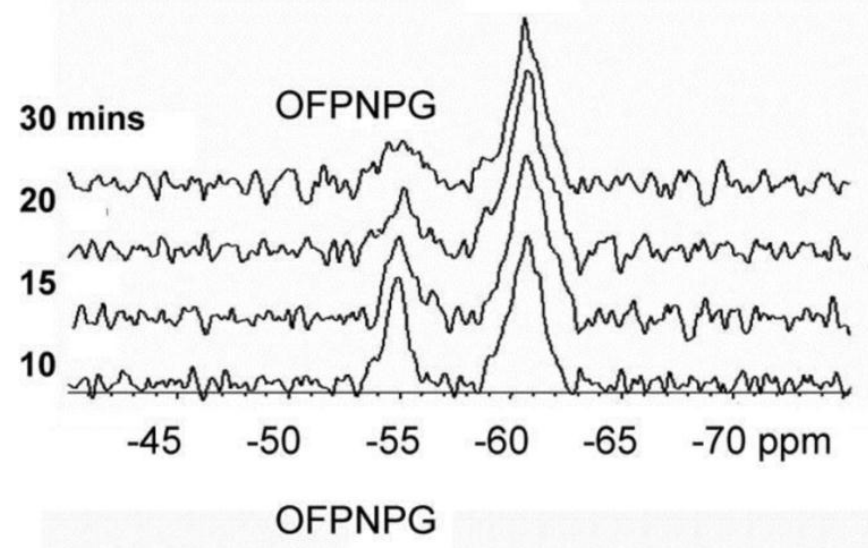

No OFPNP signal

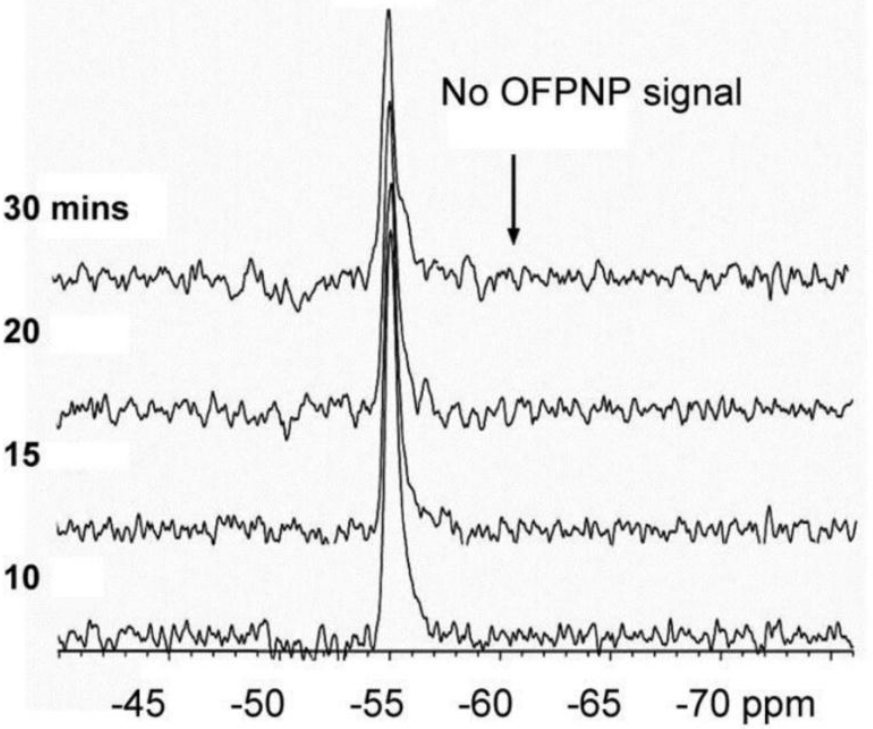

Figure 3. FI9 MR spectra MCF7 breast tumor in mice. Data demonstrating the uptake and metabolism of OFPNPG after direct intratumoral injection. The spectra in the upper panel shows the data from the lac $Z$ tumor, and the lower panel from the wild type tumor. The breakdown of OFPNPG to OFPNP was observed in the B-gal-positive tumor. No conversion was seen in the control. [Reprinted from ref. 17 with permission]. 
Another enzymatic reporter gene technique for MRS utilizes b-galactosidase. Here, 19F MRS monitors the transformation of a fluorinated version of the traditional $\quad B$-D-galactopyranoside ( $\mathrm{p}$-fluoro-onitrophenyl-B-D-galactopyranoside; PFONPG, or its isomer o-fluoro-p-nitrophenyl-B-D-galactopyranoside: OFPNPG), to aglycone (p-fluoro-o-nitrophenol: PFONP), by cells transfected with the lacZ gene. The substrate PFONPG may be superior to EgadMe since it penetrates the cell membrane to be accessible to the intracellular enzyme, thus alleviating the delivery barrier issue and facilitating the in-vivo translation [15-17].

The limitations of MRS are the kinase-based study relies on energy metabolism, and that its images are of relatively low spatial resolution. It remains to be seen whether such a reporter gene method can be translated to multicellular organisms or to multidimensional imaging $[9,18,19]$.

\section{Iron-related Reporter Gene Studies}

Recent reports have described iron-binding proteins other than metallomelanin. Weissleder et al. used an engineered transferrin receptor (ETR, Figure 4), which generated contrast by the receptor-mediated internalization of ironbound transferrin. This technique was used to image ETR-transfected (ETR+) gliosarcoma xenografts in mice after the systemic injection of a receptor-targeted probe, and it was found that ETR+ tumors showed significantly lower signal intensity on T2 gradient-echo images than control-transfected (ETR-) tumors [20]. However, the overexpression of ETR or tyrosinase could lead to iron-catalyzed free radical formation via the Fenton reaction, and be potential toxic to cells [21, 22].

Ferritin is a ubiquitous protein which stores and releases the iron, and is activated by excess free intracellular iron to maintain iron homeostasis. The pioneering work by Cohen et al was based on the hypothesis that overexpression of ferritin would trap excess intracellular iron, and provide a signal without injection of exogenous contrast agent. It was shown that the induced expression of ferritin caused significant $\mathrm{T} 2$ relaxation time shortening [23]. In a C6 glioma xenografts transfected with TET-responsive enhanced green fluorescent protein (EGFP) and heavy-chain transferrin (C6-TET-EGFP-HA-ferritin), the absence of tetracycline in drinking water was found to increase the spin-spin relaxation rate (Figure 5, 6). It should be noted that exogenous iron, such as SPIO (superparamagnetic iron oxide), was not administered for the signal detection. Genove et al. described the effect of the injection of viral vector carrying ferritin into mouse brain. Localized ferritin overexpression was found to result in decreased T2 time and thereby generating MR contrast by increasing $\mathrm{R} 1$ and $\mathrm{R} 2$ ' $(\mathrm{R} 1=1 / \mathrm{T} 2)$. It was also found that ferritin-transduced cells showed no adverse effect over control cells [24]. In fact, various reports, including a review of ferritin by Arozio et al, have suggested that ferritin plays key roles in the following process: the reduction of reactive oxygen species levels, anti-angiogenesis, and in the reduction of cellular apoptosis from oxidative stress [25-27].

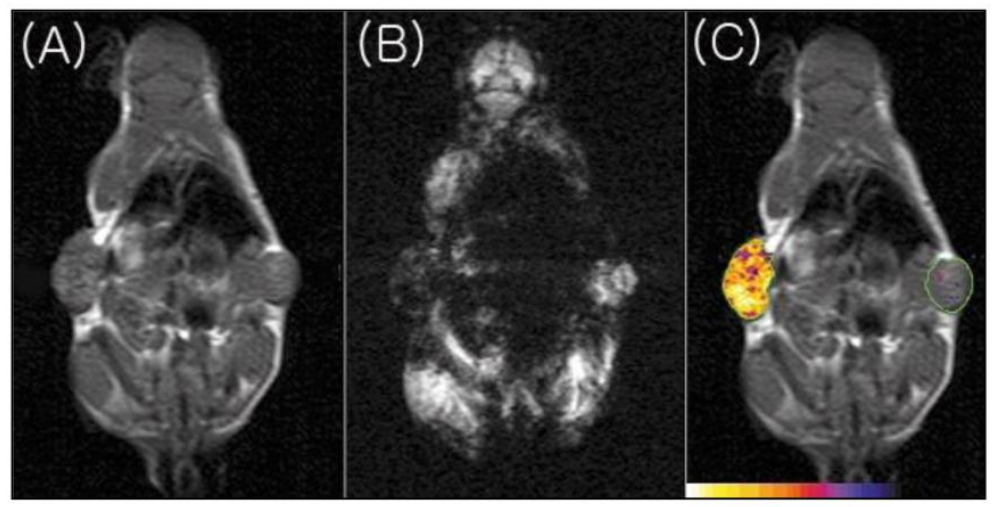

Figure 4. In vivo $M R$ imaging of a mouse engineered transferrin receptor positive (ETR+) (left image) and ETR- (right) tumors. (A) On TI-weighted images, ETR- and ETR+ tumors had similar signal intensities. (B) T2-weighted gradient-echo image showing substantial differences between ETR- and ETR+ tumors. ETR-mediated cellular accumulation of the Tf-MION probe decreases signal intensity, due to an increased transverse relaxation rate (R2) after cellular internalization, which was most pronounced using T2-and T2*-weighted sequences. (C) Composite color mapimage of a TI-weighted spin-echo obtained for anatomic detail with superimposed R2 changes after probe administration. [Reprinted from ref. 20 with permission]. 


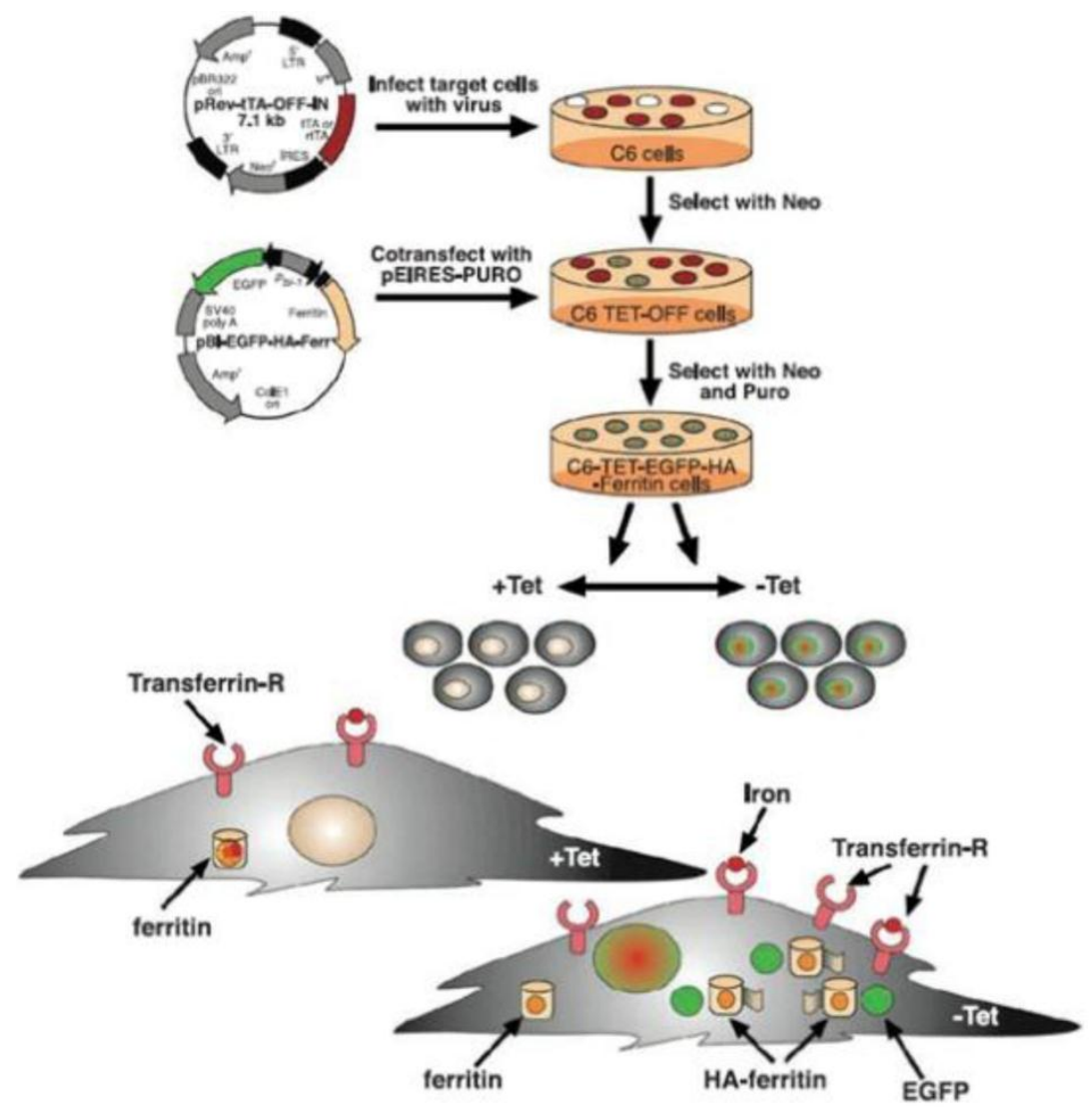

Figure 5. Schematic diagram of the expression of EGFP-HA-ferritin for multimodality endogenous reporter gene expression. C6-TET-EGFP ferritin was generated by infecting $\mathrm{C} 6$ cells with virus carrying the TET transactivator (tTA) under a constitutive promoter (pRev-tTA-OFF-IN vector). Cells were then transfected to express TET-EGFP-HA-ferritin using a bidirectional vector (pBI-EGFP-HA-Ferr vector). Selected clones showing the overexpressions of EGFP and HA-tagged ferritin, both of which were tightly suppressed by the administration of TET (+Tet).In the absence of TET (-Tet), ferritin overexpression led to the redistribution of intracellular ferritin iron and the chelation of intracellular free iron, thereby generating MR contrast by increasing $\mathrm{RI}(\mathrm{I} / \mathrm{TI})$ and $\mathrm{R} 2$. [Reprinted from ref. 23 with permission].
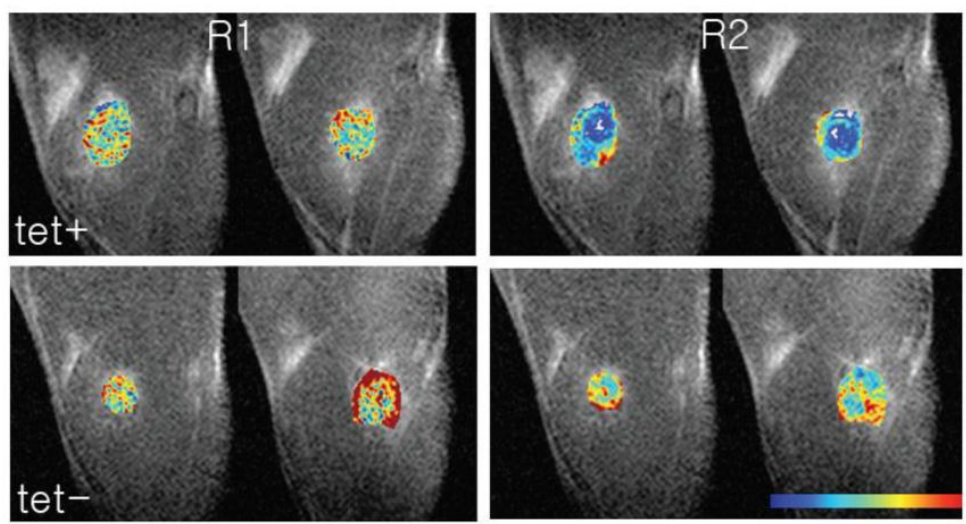

Figure 6. In vivo MR images of switchable ferritin expression in C6 tumors. RI and R2maps overlaid on the MR images of ferritin-expressing tumors. After inoculation of C6-TETEGFP-HA-ferritin tumor cells in the hind limb of nude mice, TET and sucrose (or sucrose only for control) were supplied in drinking water. (a) The images in the top row show RI and R2 in a TET+ status, and the bottom row RI and R2 in a TET- status. [Reprinted from ref. 23 with permission]. 
Questions regarding anomalies of ferritin-related relaxation remain to be answered. The phenomenon of T2-weighted image darkening by iron near protons is well known, but the measurements of relaxation rates and of iron quantities are sensitive to experimental protocols and researchers, for example, they are affected by different tissues, different solutions, molecular aggregation, MR field strength, and the quantification method used [28]. One unique feature of MRI is the linear relationship between R2 and the external magnetic field; although a quadratic relationship has been suggested $[29,30]$. For example, the relaxation rate induced by deoxyhemoglobin, another iron-associated protein, shows a quadratic increase with respect to R2. To complicate the story further, the relaxation of iron, normalized by iron concentration, has different values in different tissues and even in the same tissues from different researchers. These variances suggest that relaxation is not caused by iron
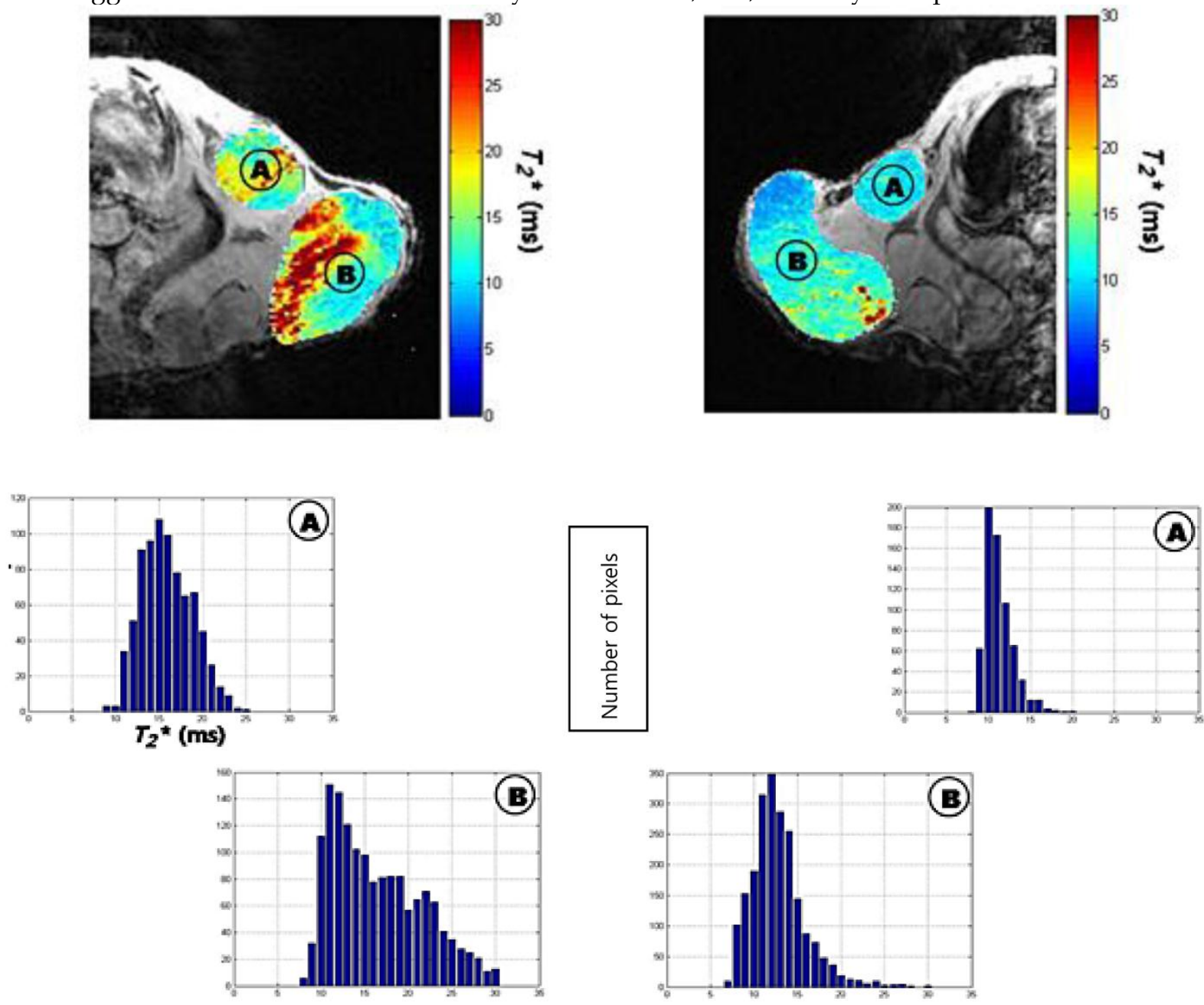

Figure 7. In vivo imaging and histological analysis of metastatic cells expressing myc-tagged human ferritin heavy chain (myc-hFTH) in lymph nodes (LNs). (A) T2* map and histograms of metastasis from myc-hFTH (right image) and control cells (left image) in the right and left axillary (A) and brachial (B) LNs in nude mice, respectively. The ferritin-carrying lymph node (white arrows in right) metastasis shows left-shift of the T2* histogram (right top and bottom) compared with the control (left top and bottom). [Reprinted from ref. 32 with permission]. 
Recently, the MagA gene, which synthesizes magnetosomes in some bacteria, has attracted research attention. The gene product has properties in common with SPIO nanoparticles. In a study by $\mathrm{Zu}$ kiya et al., the $\mathrm{T} 2{ }^{*}$-weighted images of magA-positive cells showed significant signal drops as compared with those of control cells. Electron microscopy revealed uniform particles magnetosomes. Cytosolic G6PD and MTT assays did not show cytotoxicity or change in cell proliferation. Particles produced in the magA-transfected animal cell do not cause immune response trigger on cell surfaces (Figure 8, 9). Accordingly, it has been suggested that magA-based imaging may provide a new means of noninvasive cellular tracking [33].

\section{Chemical Exchange Saturation Transfer}

The newest class of MR contrast agents utilize a process called chemical-exchange saturation transfer (CEST). Here, frequency-selective irradiation, or saturation is used to label protons noninvasively in lysine-rich protein (LRP). The spin saturation is then transferred to bulk water resonance, and thus, changes the signal intensity of the water and provides on and off contrast. Gilad et al. transfected LRP-encoding mammalian vector into glioma cells, which were then inoculated into murine brains. LRP-expressing cells were selective saturated at the exchangeable proton-resonance frequency, and the signal intensity-difference map obtained was found to differentiate between LRP-expressing tumors and control tumors using a high field magnet (11.7 Tesla). Furthermore, tumor xenografts extracted at 10 days after inoculation showed LRP gene expression (Figure 10) [34]. These results show that CEST imaging to detect gene expressions by MR, but energy deposition in tissue (specific absorption rate : SAR) and the millimolar sensitivity of the technique are limitations [35]. SAR could be described as the rate at which energy is absorbed by the body when exposed to a radiofrequency electromagnetic field, which is sufficient to cause tissue heating. Furthermore, SAR is dependent on magnetic field strength, which implies that the ultra-high magnet field needed for CEST signal detection is likely to cause significant tissue heating [36]. Further studies are required to validate the applicability of CEST-MRI in a clinical setting.

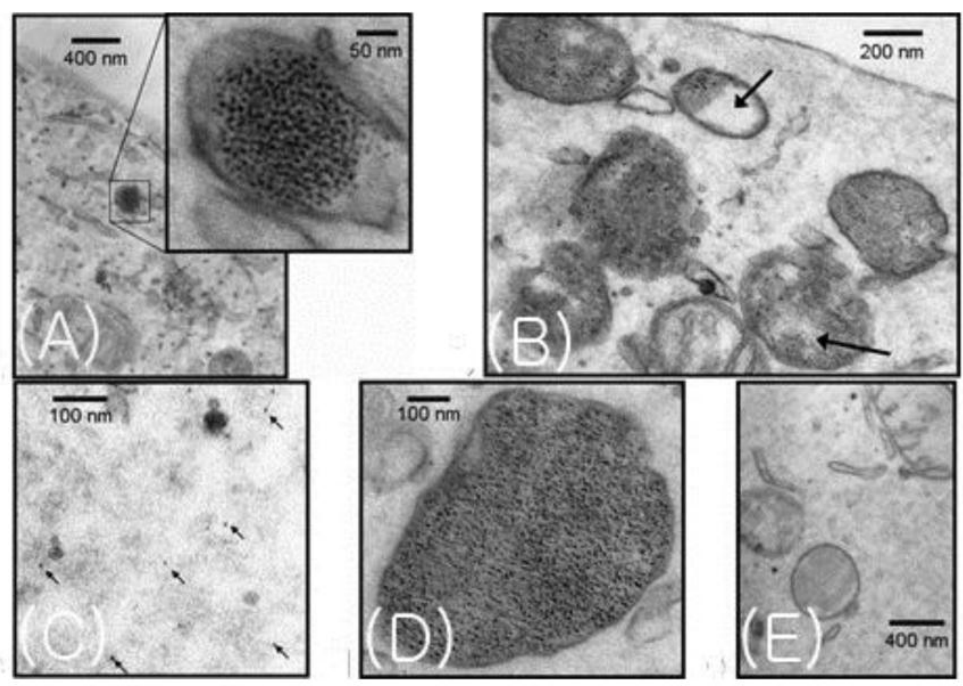

Figure 8. TEM images of magA-transduced cells. Nanoparticles were observed enclosed within membranevesicles. These vesicles, resembling endosomes, were found individually $(A)$ or in groups $(B)$, with varying numbers of particles $(B, D)$, whereas in uninduced cells (E) no particles were observed. (B) The Arrows show compartmentalization resembling the multivesicular bodies observed during cellular degradation. (C) Particles can also be seen outside the vesicles. [Reprinted from ref. 33 with permission]. 


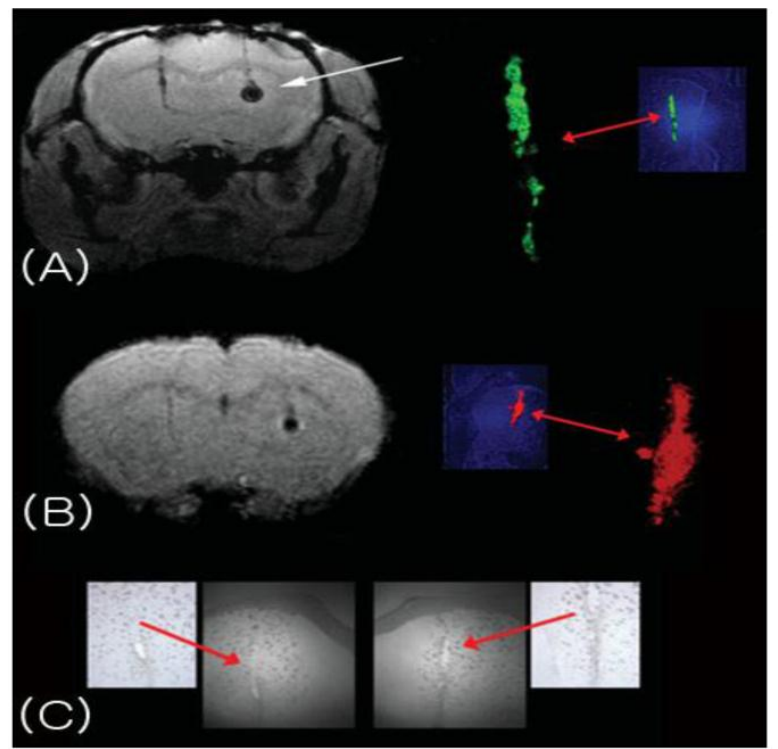

Figure 9. MRI of magA cell induction in vivo. (A) T2*-weighted image of mouse brain with transplanted magA cells (right, white arrow) and GFP control cells (left). These cells were not induced or incubated with iron supplement prior to transplantation. MagA cells exhibited significantly lower MR signals, reflecting an increase in R2, and suggesting that magA cells are able to use endogenous iron sources. The control cells on the left do not show this effect. (B) MR image of the same mouse brain showing that magA cells are readily seen by T2WI, which is less sensitive than T2* for magnetic nanoparticles. Fluorescence histology confirmed the presence of control (green) and magA-positive (red) cells. Magnified green and red channels are also shown. (C) Brightfield histology section of the brain, indicating the site of transplanted cells. [Reprinted from ref. 33 with permission].
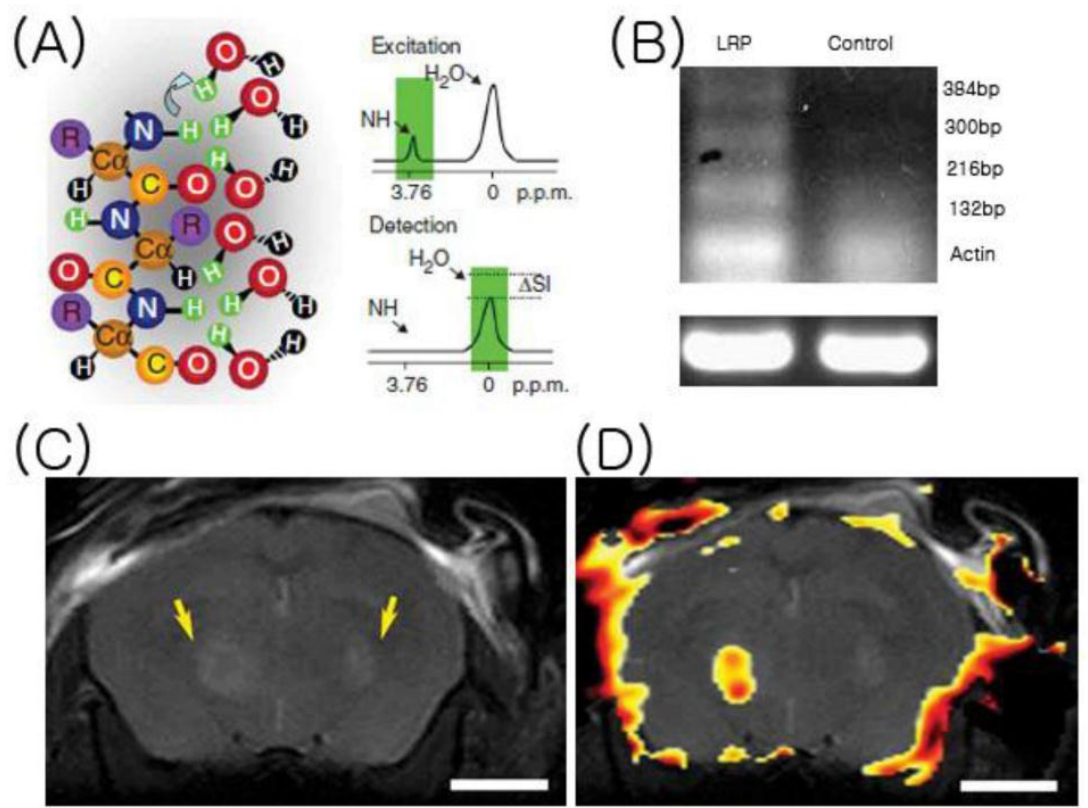

Figure 10. Chemical-Exchange Saturation Transfer. (A) Frequency-selective radiofrequency pulses label amide protons (green). These protons exchange with water protons (curved arrow), and thus, reduce MR signal intensity $(\triangle \mathrm{SI})$. (B) RT-PCR result of xenografts. (C) MR images. (D) A CEST signal intensity-difference map overlaid on anatomical images of LRP-expressing (left) and control (right) xenograft-containing brain. [Reprinted from ref. 34 with permission]. 


\section{Conclusion and future perspectives}

Progress toward the development of a good MR reporter continues to be made. MRI continues to lag behind other molecular imaging modalities in this field because of its intrinsic sensitivity and the associated necessity of high-field magnet for signal detection, because of immunologic and biologic issues, or simply because it does not provide an instant 'hot-spot' signal like PET or bioluminescence. Until recently, no report on therapeutic gene delivery mediated by a solitary imaging-and-suicide reporter, such as HSV1-sr39tK for PET imaging, had been reported for MRI [37, 38]. But researchers have showed that MR reporter gene may provide tumor-specific cytoxicity, for example, by cytosine deaminase and its metabolite 5-FU. Some approaches alleviate the need for external contrast injection, such as ferritin-related MRI, implicating its application in brain with blood-brain barrier [39]. MR is indeed slowly gaining its foothold in the field of reporter gene imaging. There is no single imaging molecular imaging modality that will meet every purpose. Fusion reporters and fusion imaging modalities will probably be devised to take advantage of the three-dimensional microanatomical imaging offered by MRI, and the combined biologic and chemical efforts being made will undoubtedly accelerate the application of molecular genetic MRI.

\section{Competing Interests}

The authors have declared that no competing interest exists.

\section{References}

1. Massoud TF, Gambhir SS. Molecular imaging in living subjects: seeing fundamental biological processes in a new light. Genes Dev. 2003; 17: 545-80.

2. Hasegawa S, Furukawa T, Saga T. Molecular MR imaging of cancer gene therapy: ferritin transgene reporter takes the stage. Magn Reson Med Sci. 2010;9: 37-47.

3. Bulte JW, Kraitchman DL. Iron oxide MR contrast agents for molecular and cellular imaging. NMR Biomed. 2004; 17: 484-99.

4. Wu JC, Abraham MR, Kraitchman DL. Current perspectives on imaging cardiac stem cell therapy. J Nucl Med. 2010;51 Suppl 1: 128S-36S.

5. Louie AY, Huber MM, Ahrens ET, Rothbacher U, Moats R, Jacobs RE, et al. In vivo visualization of gene expression using magnetic resonance imaging. Nat Biotechnol. 2000; 18: 321-5.

6. Cui W, Liu L, Kodibagkar VD, Mason RP. S-Gal, a novel $1 \mathrm{H}$ MRI reporter for beta-galactosidase. Magn Reson Med. 2010;64: 65-71.

7. Enochs WS, Petherick P, Bogdanova A, Mohr U, Weissleder R. Paramagnetic metal scavenging by melanin: MR imaging. Radiology. 1997; 204: 417-23.

8. Alfke H, Stoppler H, Nocken F, Heverhagen JT, Kleb B, Czubayko $F$, et al. In vitro MR imaging of regulated gene expression. Radiology. 2003; 228: 488-92.
9. Gilad AA, Winnard PT, Jr., van Zijl PC, Bulte JW. Developing MR reporter genes: promises and pitfalls. NMR Biomed. 2007; 20: 275-90.

10. Vande Velde G, Baekelandt V, Dresselaers T, Himmelreich U. Magnetic resonance imaging and spectroscopy methods for molecular imaging. Q J Nucl Med Mol Imaging. 2009; 53: 565-85.

11. Walter G, Barton ER, Sweeney HL. Noninvasive measurement of gene expression in skeletal muscle. Proc Natl Acad Sci U S A. 2000; 97: 5151-5.

12. Xing L, Deng X, Kotedia K, Ackerstaff E, Ponomarev V, Clifton Ling $C$, et al. Non-invasive molecular and functional imaging of cytosine deaminase and uracil phosphoribosyltransferase fused with red fluorescence protein. Acta Oncol. 2008; 47: 1211-20.

13. Stegman LD, Rehemtulla A, Beattie B, Kievit E, Lawrence TS, Blasberg RG, et al. Noninvasive quantitation of cytosine deaminase transgene expression in human tumor xenografts with in vivo magnetic resonance spectroscopy. Proc Natl Acad Sci U S A. 1999; 96: 9821-6.

14. Waerzeggers $Y$, Monfared P, Viel T, Winkeler A, Voges J, Jacobs $\mathrm{AH}$. Methods to monitor gene therapy with molecular imaging. Methods. 2009; 48: 146-60.

15. Cui W, Otten P, Li Y, Koeneman KS, Yu J, Mason RP. Novel NMR approach to assessing gene transfection: 4-fluoro-2-nitrophenyl-beta-D-galactopyranoside as a prototype reporter molecule for beta-galactosidase. Magn Reson Med. 2004; 51: 616-20.

16. Yu J, Otten P, Ma Z, Cui W, Liu L, Mason RP. Novel NMR platform for detecting gene transfection: synthesis and evaluation of fluorinated phenyl beta-D-galactosides with potential application for assessing LacZ gene expression. Bioconjug Chem. 2004; 15: 1334-41.

17. Yu JX, Kodibagkar VD, Liu L, Mason RP. A 19F-NMR approach using reporter molecule pairs to assess beta-galactosidase in human xenograft tumors in vivo. NMR Biomed. 2008; 21: 704-12.

18. Koretsky AP, Traxler BA. The B isozyme of creatine kinase is active as a fusion protein in Escherichia coli: in vivo detection by 31P NMR. FEBS Lett. 1989; 243: 8-12.

19. Ki S, Sugihara F, Kasahara K, Tochio H, Okada-Marubayashi A, Tomita S, et al. A novel magnetic resonance-based method to measure gene expression in living cells. Nucleic Acids Res. 2006; 34: e51.

20. Weissleder R, Moore A, Mahmood U, Bhorade R, Benveniste $H$, Chiocca EA, et al. In vivo magnetic resonance imaging of transgene expression. Nat Med. 2000; 6: 351-5.

21. Arredondo M, Nunez MT. Iron and copper metabolism. Mol Aspects Med. 2005; 26: 313-27.

22. Kotamraju S, Chitambar CR, Kalivendi SV, Joseph J, Kalyanaraman B. Transferrin receptor-dependent iron uptake is responsible for doxorubicin-mediated apoptosis in endothelial cells: role of oxidant-induced iron signaling in apoptosis. J Biol Chem. 2002; 277: 17179-87.

23. Cohen B, Dafni H, Meir G, Harmelin A, Neeman M. Ferritin as an endogenous MRI reporter for noninvasive imaging of gene expression in C6 glioma tumors. Neoplasia. 2005; 7: 109-17.

24. Genove G, DeMarco U, Xu H, Goins WF, Ahrens ET. A new transgene reporter for in vivo magnetic resonance imaging. Nat Med. 2005; 11: 450-4.

25. Arosio P, Ingrassia R, Cavadini P. Ferritins: a family of molecules for iron storage, antioxidation and more. Biochim Biophys Acta. 2009; 1790: 589-99.

26. Pham CG, Bubici C, Zazzeroni F, Papa S, Jones J, Alvarez K, et al. Ferritin heavy chain upregulation by NF-kappaB inhibits TNFalpha-induced apoptosis by suppressing reactive oxygen species. Cell. 2004; 119: 529-42. 
27. Cozzi A, Corsi B, Levi S, Santambrogio P, Albertini A, Arosio P. Overexpression of wild type and mutated human ferritin H-chain in HeLa cells: in vivo role of ferritin ferroxidase activity. J Biol Chem. 2000; 275: 25122-9.

28. Gossuin Y, Muller RN, Gillis P. Relaxation induced by ferritin: a better understanding for an improved MRI iron quantification. NMR Biomed. 2004; 17: 427-32.

29. Wood JC, Fassler JD, Meade T. Mimicking liver iron overload using liposomal ferritin preparations. Magn Reson Med. 2004; 51: 607-11.

30. Cohen B, Ziv K, Plaks V, Israely T, Kalchenko V, Harmelin A, et al. MRI detection of transcriptional regulation of gene expression in transgenic mice. Nat Med. 2007; 13: 498-503.

31. Gossuin Y, Gillis P, Muller RN, Hocq A. Relaxation by clustered ferritin: a model for ferritin-induced relaxation in vivo. NMR Biomed. 2007; 20: 749-56.

32. Choi SH, Cho HR, Kim HS, Kim YH, Kang KW, Kim H, et al. Imaging and quantification of metastatic melanoma cells in lymph nodes with a ferritin MR reporter in living mice. NMR Biomed. 2011; doi: 10.1002/nbm.1788.

33. Zurkiya O, Chan AW, Hu X. MagA is sufficient for producing magnetic nanoparticles in mammalian cells, making it an MRI reporter. Magn Reson Med. 2008; 59: 1225-31.

34. Gilad AA, McMahon MT, Walczak P, Winnard PT, Jr., Raman $\mathrm{V}$, van Laarhoven $\mathrm{HW}$, et al. Artificial reporter gene providing MRI contrast based on proton exchange. Nat Biotechnol. 2007; 25: $217-9$

35. Aime S, Castelli DD, Crich SG, Gianolio E, Terreno E. Pushing the sensitivity envelope of lanthanide-based magnetic resonance imaging (MRI) contrast agents for molecular imaging applications. Acc Chem Res. 2009; 42: 822-31.

36. Schick F. Whole-body MRI at high field: technical limits and clinical potential. Eur Radiol. 2005; 15: 946-59.

37. Penuelas I, Haberkorn U, Yaghoubi S, Gambhir SS. Gene therapy imaging in patients for oncological applications. Eur J Nucl Med Mol Imaging. 2005; 32 Suppl 2: S384-403.

38. Yaghoubi SS, Barrio JR, Namavari M, Satyamurthy N, Phelps ME, Herschman HR, et al. Imaging progress of herpes simplex virus type 1 thymidine kinase suicide gene therapy in living subjects with positron emission tomography. Cancer Gene Ther. 2005; 12: 329-39.

39. Lelyveld VS, Atanasijevic T, Jasanoff A. Challenges for Molecular Neuroimaging with MRI. Int J Imaging Syst Technol. 2010;20: 71-9. 